$\mathrm{g}_{2}$ gene on long-term weight change in Finnish non-diabetic subjects. Diabetologia 44: 925-926

2. Stumvoll M, Wahl HG, Löblein K et al. (2001) The Pro12Ala polymorphism in the peroxisome proliferator activated receptor $\mathrm{g} 2$ gene is associated with increased antilipolytic insulin sensitivity. Diabetes 50: 876-881

3. Stumvoll M, Wahl HG, Löblein K et al. (2001) A novel use of the hyperinsulinemic-euglycemic clamp technique to measure insulin sensivity of systemic lipolysis. Horm Metab Res 33: 89-95

4. Altshuler D, Hirschhorn JN, Klannemark Met al. (2000) The common PPAR gamma Pro12Ala polymorphism is associated with decreased risk of type 2 diabetes. Nat genet 26: 76-80

5. Reitman ML, Arioglu E, Gavrilova O, Taylor SI (2000) Lipoatrophy revisited. Trends Endocrinol Metab 11: 410-416

\section{A novel hepatocyte nuclear factor-1及 (MODY-5) gene mutation in an Italian family with renal dysfunctions and early-onset diabetes}

To the Editor: Mutations in the homeodomain-containing transcription factor hepatocyte nuclear factor $(H N F)-1 \beta$ have been reported in association with maturity-onset diabetes of the young (MODY-5) and early-onset and progressive non-diabetic renal dysfunction [1-2]. Iwasaki et al. recently screened the $H N F-1 \beta$ gene for mutations in a Japanese family whose members had $H N F-1 \beta$ diabetes, renal dysfunction and abnormalities of genital development [3]. They found a new heterozygous mutation in the hepatocyte nuclear factor $(H N F)-1 \beta$ located in the splice donor site of exon 2 , associated with these different clinical phenotypes. In addition, the results of Weng et al. suggested that $H N F-1 \beta$ mutations might play an important role in the development of renal disease [4].

Here, we report a three-generation Italian family in which a syndrome of diabetes and severe non-diabetic renal disease is associated with a novel deletion mutation in the $H N F-1 \beta$ gene (Fig. 1).

The index subject (III-1) was a girl with polycystic kidneys who was followed from the birth. During follow-up the patient showed incidental hyperglycaemia and impaired glucose tolerance on OGTT, at 12 years of age. After kidney transplantation, at 13 years of age she developed diabetes and insulin therapy started.

The index subject's two younger sisters (III-2 and III-3) had small kidneys with occasional cysts and a normal glucose tolerance test.

Her father (II-2) had horseshoe kidney and after renal surgical removal chronic renal insufficiency (CRI) developed. When the father was 20 years old, he was diagnosed as having diabetes and was treated with insulin.

All these subjects underwent immunologic (islet cell autoantibodies, such as IAA, GADA and IA-2A) and immunogenetic (HLA typing) markers for Type I (insulin-dependent) diabetes mellitus. No autoantibodies were present in the subjects; regarding DQA1 and DQB1 typing, one heterodimer was present in the two sisters and in the father, no heterodimers were in the index subject.

Moreover the paternal aunt (II-1) and grandmother (I-2) of the index subject showed renal dysplasia with renal insufficiency without history of diabetes.

Therefore, renal manifestations are variable but are a constant feature in all six members of this family; until now only
6. Kubota N, Terauchi Y, Miki H et al. (1999) PPAR gamma mediates high-fat diet-induced adipocyte hypertrophy and insulin resistance. Mol Cell 4: 597-609

7. Deeb SS, Fajas L, Nemoto M et al. (1998) A Pro12Ala substitution in PPARgamma2 associated with decreased receptor activity, lower body mass index and improved insulin sensitivity. Nat Genet 20: 284-287

8. Adams M, Montague CT, Prins JB et al. (1997) Activators of peroxisome proliferator-activated receptor gamma have depot-specific effects on human preadipocyte differentiation. J Clin Invest 100: 3149-3153

Corresponding author: M. Stumvoll, Medizinische Universitätsklinik, Otfried-Müller-Str. 10, Tübingen, Germany, e-mail: michael.stumvoll@med.uni-tuebingen.de

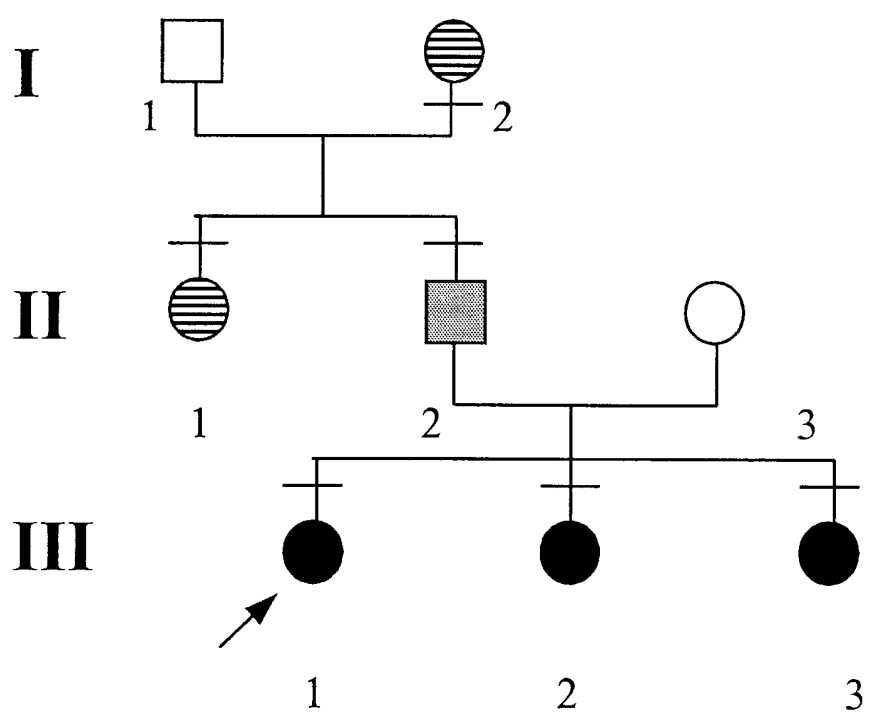

Fig. 1. A family tree showing clinical details and inheritance of $H N F-1 \beta$ mutation. The index subject (III-1), with bilateral renal cysts and diabetes diagnosed at 13 years of age, is indicated by an arrow. The proband's father (II-2) with horseshoe kidney and diabetes diagnosed at 20 years is represented by a grey square. Heterozygous mutation HNF-1ß (black line); Horseshoe kidney (solid square); Bilateral renal cysts (solid circle); Displastic kidneys (striped circle)

two had early onset diabetes, diagnosed before 25 years of age, without immunologic and immunogenetic markers for Type I diabetes susceptibility.

DNA was isolated from peripheral blood from these six patients in order to evaluate the $H N F-1 \beta$ gene.

The minimal promoter and coding regions of the nine exons and flanking introns of the $H N F-1 \beta$ gene were screened for mutations by direct sequencing of PCR products using specific primers as described [1]. The novel mutation was confirmed by cloning the PCR product into the vector pCR2.1TOPO (Invitrogen, Carlsbad, Calif., USA) and sequencing clones derived from both alleles.

We found an heterozygous deletion in the splice donor site of intron 2 of the $H N F-1 \beta$ gene, located after the conserved GT-dinucleotide (designated IVS2 + 2delaagt), which might result in abnormal splicing of HNF- $1 \beta$ mRNA. This issue is currently under investigation and functional studies on the DNA transcripts and the encoded HNF- $1 \beta$ protein are in progress. 
This mutation was identified with renal dysfunction in all six affected patients in a dominant way of inheritance over three generations and was never observed in 100 control chromosomes.

Our study represents the first Italian molecular characterization of a MODY-5 family and further confirm that mutations in $H N F-1 \beta$ gene are associated with a syndrome characterized by MODY and kidney disease.

The presence of this splice site $H N F-1 \beta$ mutation support previously reported results [3], suggesting that alterations in the conserved sequence elements at the splice donor site of intron 2 might be a hot spot region for mutations in the $H N F-1 \beta$ gene.

Acknowledgements. This work was supported by grants from the Italian Ministry of Health (Ricerca Corrente - G.Gaslini Institute, 1999) and Telethon-Italy 1999.

I. Carbone, M. Cotellessa, C. Barella, C. Minetti, G. M. Ghiggeri, G. Caridi, F. Perfumo, R. Lorini

\section{Identification of a gain-of-function mutation in the HNF-1及 gene in a Japanese family with MODY}

To the Editor: Mutations in the hepatocyte nuclear factor $(H N F)-1 \beta$ gene cause maturity-onset diabetes of the young (MODY5) [1]. Clinical examinations of patients with the mutations have shown MODY5 to be associated with progressive renal dysfunction and bilateral renal cysts as well. Three of the seven mutations so far reported have been identified in Japanese [1-6]. In order to ascertain the prevalence of $H N F$ $1 \beta$ mutations in early-onset Type II (non-insulin-dependent) diabetes mellitus in Japanese more precisely, we screened a larger group of 111 Japanese patients with early-onset Type II diabetes for mutations in the $H N F-1 \beta$ gene. Ten of the patients were classified as having MODY, with Type II diabetes in at least three generations consistent with autosomal dominant transmission and onset of the disease in patients before 25 years of age. The study group also includes 85 non-obese $\left(\mathrm{BMI}<25 \mathrm{~kg} / \mathrm{m}^{2}\right)$ patients with Type II diabetes diagnosed before 15 years of age because our previous study of MODY3 in young Japanese patients suggested that many of these patients have a monogenic form of diabetes [7]. The other sixteen patients were diagnosed with diabetes before 35 years of age and had a family history of diabetes among first-degree relatives. The minimal promoter, coding regions and flanking introns of the $H N F-1 \beta$ gene were directly sequenced in these patients, resulting in the identification of a novel missense mutation (TCC to TTC: S36F) in the heterozygous state in one MODY family. The serine residue at codon 36 is conserved in human, mouse, and rat $H N F-1 \beta$. This nucleotide alteration was not found in any of the 100 Japanese control subjects (200 alleles).

The subject with the S36F mutation is a 46-year-old female who was diagnosed with Type II diabetes at 17 years of age. She also has proteinuria and two cysts (diameter; $6.9 \mathrm{~cm}$ and $8.9 \mathrm{~cm}$ ) in the left kidney. Her serum creatinine concentration is normal $(0.6 \mathrm{mg} / \mathrm{dl}$; normal 0.6 to $1.0 \mathrm{mg} / \mathrm{dl})$ at present. Genotyping of other family members with diabetes was done (Fig. 1A). The mutation was found in the subject's elder daughter who was diagnosed with Type II diabetes at 14 years

\section{References}

1. Horikawa Y, Iwasaki N, Hara M et al. (1997) Mutation in hepatocyte nuclear factor- $1 \beta$ gene (TCF2) associated with MODY. Nat Genet 17: 384-385

2. Nishigori H, Yamada S, Kohama T et al. (1998) Frameshift mutation, A263fsinsGG, in the hepatocyte nuclear factor$1 \beta$ gene associated with diabetes and renal dysfunction. Diabetes 47: 1354-1355

3. Iwasaki N, Okabe I, Momoi MY, Ohashi H, Ogata M, Iwamoto Y (2001) Splice site mutation in the hepatocyte nuclear factor- $1 \beta$ gene, IVSnt $+1 \mathrm{G}>\mathrm{A}$, associated with maturity-onset diabetes of the young, renal dyplasia and bicornuate uterus. Diabetologia 44: 387-391

4. Weng JP, Lehto M, Forsblom C, Huang X, Li H, Groop LC (2000) Hepatocyte nuclear factor- $1 \beta$ (MODY-5) gene mutations in Scandinavian families with early-onset diabetes or kidney disease or both. Diabetologia 43: 131-132

Corresponding author: R. Lorini ( $\bowtie)$ Department of Pediatrics, University of Genova, G. Gaslini Institute, Italy

of age $(\mathrm{BMI}=22.9)$ and is being treated with insulin. The same mutation was also present in the 68-year-old mother, who was diagnosed with Type II diabetes around 60 years of age. However, interestingly, the S36F mutation was not identified in the other 17-year-old daughter $(\mathrm{BMI}=20.3)$, whose glycosuria was noticed at 11 years of age by an annual health examination, suggesting that other diabetogenic factors contributed to the development of diabetes in this subject.

In order to determine whether the mutation identified in this family alters the function of the HNF- $1 \beta$ protein, the effect of the wild-type and mutant $H N F-1 \beta$ on transcription from three different promoters of the target genes was examined. HeLa cells were transfected with $500 \mathrm{ng}$ of pcDNA3.1-wild type (WT)-HNF-1 $\beta$ or pcDNA3.1-S36F-HNF-1 $\beta$ expression vector with L-type pyruvate kinase (PKL) and transthyretin (TTR) promoter luciferase reporter genes (Fig.1B). The transactivation ability of S36F-HNF-1 $\beta$ was significantly increased by $45.3 \%$ (PKL: $p=0.028$ ) and by $56.7 \%$ (TTR: $p=0.022$ ) compared with WT-HNF-1 $\beta$. The HNF-1 $\beta$ up-regulates GLUT2 gene expression in mouse insulin-secreting MIN6 cells [2]. A significant increase in the transactivation activity of S36F-HNF-1 $\beta$ compared with WT-HNF-1 $\beta$ also was observed in these cells $(p=0.005)$. S36F-HNF-1 $\beta$ (25-1000 ng) consistently showed $12-57 \%$ higher activity on TTR and GLUT2 reporters compared WT-HNF-1 $\beta$ and the activity was significantly increased at high concentrations (100 ng- $1 \mu \mathrm{g}$ ). These results suggest that $\mathrm{S} 36 \mathrm{~F}$ is a gain-of-function mutation. We previously reported that $\mathrm{HNF}-1 \beta$ mutations impair pancreatic beta-cell function by loss-of-function and dominant-negative mechanisms [2]. Gain-of-function could be a third mechanism, although its pathogenesis of impaired insulin secretion is not known. Recently, it has been reported that a frameshift mutation (P328L329fsdelCCTCT), identified in a European MODY5 family with renal abnormalities, acts as a gain-of-function mutation ( twofold) [8]. Thus, S36F mutation in the $H N F-1 \beta$ gene might affect the clinical phenotypes in this MODY family.

In conclusion, we have identified a novel missense mutation in the $H N F-1 \beta$ gene in a Japanese family with MODY but the significance of the mutation in the development of diabetes in the family is not clear. The present results together with previous ones $(1,2,6$ and this study) indicate that $H N F$ - 